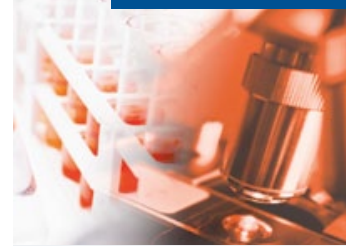

Berichte aus der Pharmaindustrie
1. Lukashevich et al DOM 2011:13:947-54

\title{
Orale Diabetestherapie Gliptin jetzt auch in Monotherapie und bei Nierenschwäche
}

- Etwa ein Viertel aller Typ-2-Diabetiker kann Metformin aufgrund von Unverträglichkeiten oder Kontraindikationen nicht einnehmen. Ihnen kann nun Vildagliptin (Galvus ${ }^{\circledast}$ ) in Monotherapie verordnet werden. Die entsprechende Zulassung der Europäischen Kommission wurde kürzlich erteilt.

Neben häufigen gastrointestinalen Nebenwirkungen ist es v.a. die mit dem Alter fortschreitende Niereninsuffizienz, die den Einsatz von Metformin in der First-line-Therapie bei Typ-2-Diabetes limitiert, sagte der Diabetologe Professor Matthias Blüher, Leipzig. Etwa die Hälfte aller Patienten über 75 Jahre sind moderat oder schwer niereninsuffizient. Sinkt die glomeruläre Filtrationsrate (GFR) unter $60 \mathrm{ml} /$ min, ist Metformin kontraindiziert. Vor diesem Hintergrund begrüßte er, dass mit Vildagliptin nun eine Alternative zur Verfügung steht.

Die Wirksamkeit und Sicherheit in dieser Indikation stellte der DPP-4-Hemmer in einer kontrollierten Doppelblindstudie mit 525 Patienten unter Beweis, von denen je die Hälfte eine moderate oder schwere Nierenschwäche hatte [1]. In beiden Kollektiven reduzierte der DPP-4-Hemmer den $\mathrm{HbA}_{1 \mathrm{c}}$-Wert im Vergleich zur Placebogruppe um 0,5 bis $0,6 \%$. Die
Rate schwerer unerwünschter Nebeneffekte, darunter schwere Hypoglykämien, unterschied sich nicht von der Placebogruppe. Blüher: Gerade in einem Hochrisiko-Patientenkollektiv mit wahrnehmbarer Mortalität ist Therapiesicherheit, v.a. ein geringes Hypoglykämie-Risiko, ein wichtiger Aspekt.

Zu den Vorteilen von Vildagliptin gehört, dass die Dosis im Alter nicht angepasst werden muss, so der in Augsburg niedergelassene Diabetologe Dr. Michael Dietlein. Bei Niereninsuffizienz mit einer GFR $<50$ $\mathrm{mg} / \mathrm{dl}$ liegt die Dosierung bei 1x50 mg/d. Innerhalb der Gruppe der DPP-4-Hemmer ist Vildagliptin laut Dietlein am stärksten wirksam. Ein weiteres Plus der Substanz: Sie wird, so der Diabetologe, nicht über CYP3A4 verstoffwechselt, was bei älteren Patienten mit Polypharmazie von Relevanz sein kann.

Vildagliptin ist in klinischen Studien mit über 15 000 Patienten untersucht worden. Die Erfahrung beträgt inzwischen über zwei Millionen PatientenBehandlungsjahre, teilt Novartis mit.

Quelle: Pressekonferenz „Monotherapie mit Vildagliptin: neue Option bei Patienten mit Niereninsuffizienz oder Metformin-Kontraindikationen, München, Februar 2012, Veranstalter: Novartis

\section{Eine Chance für Innovationen im Alltag Visualisierungstool für Blutzuckerdaten besteht großen Praxistest}

- Innovation und gute Qualität sind keine Garanten für eine rasche und breite Umsetzung von Konzepten in der medizinischen Praxis. Dies gilt auch bei der Darstellung und Verwaltung der Daten aus dem Blutzuckerselbstmanagement. Ein innovatives Visualisierungstool ist hier ein entscheidendes Stück vorangekommen, hat es sich doch offenbar in einer großen Praxisstudie eine Fangemeinde erobert.

Bei der Einführung fortschrittlicher Konzepte in den klinischen Alltag ist neben einer großen Portion Evidenz oft viel Engagement gefragt. „Dennoch muss man hier sehr bescheiden sein, das hat die Geschichte der Medizin gezeigt", wie Prof. Dr. phil. Florian Steger, Direktor des Instituts für Medizingeschichte und Ethik in Halle, bei einer Veranstaltung im Schloss Hohenkammer bei München gesagt hat. So dauerte es Jahrzehnte, bis sich die Erkenntnisse über Vorhandensein und Verbreitung von Krankheitserregern flächendeckend in effektiver Hygiene niederschlugen. Und auch in der jüngeren Medizingeschichte gibt es hierfür Beispiele. So etwa das der Entdeckung von Helicobacter pylori 1983 als Verursacher gastroduodenaler Ulzera durch Barry Marshall und John Robin Warren. „Diese Theorie wurde lange skeptisch be- trachtet und man beharrte auf der Stresshypothese mit Übersäuerung des Magens“, so Steger. Erst Jahre später wurde die Genese anerkannt und die Eradikationstherapie eingeführt. Im Jahr 2005 gab es dann den Medizinnobelpreis für Marshall und Warren.

\section{Im Diabetesmanagement mehr erkennen und mehr erreichen}

Die Intervalle von der Idee bis zur Einführung in die Praxis sind kürzer geworden, das Phänomen bleibt: Innovationen in der Medizin haben es oft schwer. Das bezieht sich auch auf die Medizintechnik. „Die Medizintechnologie ist weit fortgeschritten, aber es ist eine Herausforderung, sie in den Praxisalltag $\mathrm{zu}$ integrieren“, sagte Prof. Oliver Schnell von der Forschergruppe Diabetes am Helmholtz-Zentrum München. Man habe erkannt, dass neue Arzneien und die Fokussierung auf den $\mathrm{HbA}_{1 \mathrm{c}}$-Wert allein nicht immer zielführend sind, wenn es um eine optimale Stoffwechseleinstellung und die Lebensqualität der $\mathrm{Pa}$ tienten geht. Das individuelle Blutglukoseprofil mit seinen Höhen und Tiefen - die Glukosevariabilität - ist ebenfalls wichtig. Und hier kommt die Blutzuckerselbstmessung (BZSM) mithilfe moderner tech- 